\title{
UM ESTUDO HISTÓRICO-MATEMÁTICO ACERCA DA TESE DE DOUTORAMENTO DE THEODORO AUGUSTO RAMOS (1895-1935)
}

\author{
Sabrina Helena Bonfim \\ Universidade Federal de Mato Grosso do Sul - UFMS - Brasil \\ Marcos Vieira Teixeira \\ Universidade Estadual Paulista - UNESP - Brasil
}

(aceito para publicação em fevereiro de 2019)

\begin{abstract}
Resumo
Adentrando-se na Matemática e especificamente na História da Matemática focada no Brasil, com seus personagens e obras que possuíram notável importância para o desenvolvimento desta ciência, este artigo tem como escopo apresentar um estudo histórico-matemático acerca da tese de doutoramento do engenheiro-matemático Theodoro Augusto Ramos (1895-1935). Intitulada Sobre as funcções de variaveis reaes e datada do ano de 1918, foi defendida para obtenção do grau de doutor em Ciências Físicas e Matemáticas pela Escola Politécnica do Rio de Janeiro. O olhar dado ao tratamento do tema remeteu-se à História da Matemática nos âmbitos da Educação Matemática.
\end{abstract}

Palavras-chave: Matemática, História, Theodoro Augusto Ramos, Funções de Variáveis Reais.

\section{[A HISTORICAL-MATHEMATICAL STUDY ABOUT THE DOCTOR'S THESIS OF THEODORO AUGUSTO RAMOS (1895-1935)]}

\begin{abstract}
Entering in the Mathematics and specifically focused on History of Mathematics in Brazil, with their characters and publications that possessed remarkable importance for the development of this science, this article has the objective to present a study about the historical and mathematical PhD thesis of engineer-mathematician Theodoro Augusto Ramos
\end{abstract}


(1895-1935). Entitled Sobre as funcções de variaveis reaes and dated from 1918, it was defended for the degree of Doctor in Physical and Mathematical Sciences from the Polytechnic School of Rio de Janeiro. The look given to the treatment of the subject referred to the History of Mathematics in the fields of Mathematics Education.

Keywords: Mathematics, History, Theodoro Augusto Ramos, Functions of Real Variables.

\section{Introdução:}

“[...] Por definição, o homem de ideias se deixa ler por suas publicações, não por seu cotidiano”. (François Dosse, 2009)

Neste artigo apresenta-se um estudo histórico-matemático comentado da tese de doutoramento do engenheiro-matemático Theodoro Augusto Ramos ${ }^{1}$ (1895-1935), intitulada Sobre as funções de variáveis reaes e defendida na Escola Politécnica do Rio de Janeiro em $1918^{2}$, no que concerne ao seu tema central, demonstrações principais e contribuições, dentre outros. A escolha desse objeto de estudo se deu devido, principalmente, a originalidade da tese em questão, uma prática não comum para a época, e, sobretudo pela minuciosa pesquisa matemática realizada por este então engenheiro-matemático acerca de questões e problemas atuais de matemática para a ocasião no Brasil e no mundo.

Theodoro Augusto Ramos ${ }^{3}$ graduou-se em Engenharia Civil no ano de 1916 pela Escola Politécnica do Rio de Janeiro, embora seu interesse tenha sido pelas ciências matemáticas, até então, estudadas somente nas Escolas de Engenharia. Segundo as normas da instituição, os que assim concluíssem sua graduação poderiam submeter-se a defesa de tese e, se aprovados, teriam o grau de doutor nas mesmas ciências. Salienta-se que os cursos de Engenharia Civil, de Minas, Industrial e Mecânica conferiam grau em Bacharel em Ciências Físicas e Matemáticas (como obtido por Theodoro), ao passo que o curso de Engenharia Agronômica, oferecia grau de Bacharel em Ciências Físicas e Naturais ${ }^{4}$. Estiveram presentes em sua banca os professores catedráticos Licínio Athanásio Cardoso ${ }^{5}$ e

\footnotetext{
${ }^{1}$ Para uma leitura detalhada acerca da biografia de Theodoro Augusto Ramos, consultar o artigo da autora: Theodoro Augusto Ramos (1895-1935): uma biografia (Revista Brasileira de História da Matemática - RBHM, Vol. 14, no. 29, 2015).

${ }^{2}$ Na pesquisa utiliza-se a publicação feita pela Seção de Obras do Estado de São Paulo e também datada do ano de 1918. O exemplar consultado encontra-se nos arquivos da biblioteca da Escola Politécnica da USP.

${ }^{3}$ Dados referentes a Theodoro Augusto Ramos também podem ser encontrados nas referências do autor: SILVA, Clovis Pereira da, Início e Consolidação da Pesquisa Matemática no Brasil. Brasília: Senado Federal, Conselho Editorial, 2008. (v.98), A Matemática no Brasil. História de seu Desenvolvimento. São Paulo: Editora Edgard Blücher Ltda. 3. ed., 2003 e Theodoro A. Ramos: Sua Correspondência para Lélio Gama. Revista da SBHC, n. 17, p. 11-20, 1997.

${ }^{4}$ MILLER, Célia Peitl. O Doutorado em Matemática no Brasil: Um Estudo Histórico Documentado (1842 a 1937). Dissertação de Mestrado. Área de Concentração em Ensino e Aprendizagem da Matemática e seus Fundamentos Filosófico-Científicos. Rio Claro, SP, 2003.

${ }^{5}$ Licínio Athanásio Cardoso (1852-1926): Concluiu os estudos da Escola Militar em 1874, e o curso de Engenharia Militar em 1879, sendo nomeado no ano seguinte professor do curso preparatório. Promovido a capitão, em 1885 ,
} 
Francisco Bhering ${ }^{6}$, assim como os professores substitutos no exercício de catedráticos: Augusto de Brito Belford Roxo ${ }^{7}$ e Maurício Joppert da Silva ${ }^{8}$.

Corroborando com as palavras de Freire (1936), o mais profundo e notável trabalho de Theodoro Ramos foi, sem dúvida, a sua These de doutorado: "Sobre as funcções de variáveis reaes". É incontestável. Sobretudo se se leva em conta o facto desse trabalho ter sido por ele produzido aos 23 anos de idade.

O tema então estudado por Theodoro provocou, na época da defesa, certo estranhamento, visto não ser ainda comum para a comunidade matemática brasileira da época a realização de tais discussões, principalmente em um curso que não era de Matemática, pois até então estes não existiam no país. Segundo Gama:

"[...] Teodoro Ramos, ao fim do curso, apresenta sua tese de doutorado, Sobre Funções Reais de Variável Real. Este acontecimento criou, na Escola, uma atmosfera densa, opaca, cheia de apreensões, de parte a parte. Um jovem estudante desafiava os cânones oficiais, com uma tese estranha, um trabalho exótico [...]”. (GAMA, 1965, p.27)

\section{Aspectos matemáticos principais da tese Sobre as funções de variáveis reaes:}

Intitulada Sobre as funcções de variaveis reaes e datada do ano de 1918 a referida tese encontra-se estruturada em sete partes sendo: prefácio; introdução (dividida em os conjuntos lineares, os conjuntos a duas dimensões, a medida dos conjuntos, a noção moderna de função, a convergência das sucessões de funções, a convergência uniforme); as funções de uma variável real (onde o autor trata as funções contínuas e as funções de classe 1, dividindo em: as funções contínuas, as funções contínuas deriváveis, as funções indefinidamente deriváveis, as funções de classe I); a teoria das funções somáveis (as sucessões de Baire, a integração das funções somáveis, a representação efetiva das funções somáveis, nota sobre uma fórmula de interpolação, nota sobre a aproximação das funções duas vezes deriváveis); as funções de duas variáveis reais (tratando da representação efetiva das funções somáveis de duas variáveis reais); proposições (segue aqui uma explanação dos conteúdos das "cadeiras" vistas durante a graduação e que foram utilizadas na elaboração da tese. São elas: $1^{\circ}$ ano: $1^{\mathrm{a}}, 2^{\mathrm{a}}$ e $3^{\mathrm{a}}$ cadeiras; $2^{\circ}$ ano: $1^{\mathrm{a}}, 2^{\mathrm{a}}$ e $3^{\mathrm{a}}$ cadeiras; $3^{\circ}$ ano: $1^{\mathrm{a}}, 2^{\mathrm{a}}, 3^{\mathrm{a}}$ e $4^{\mathrm{a}}$ cadeiras; $4^{\circ}$ ano: $1^{\mathrm{a}}, 2^{\mathrm{a}}, 3^{\mathrm{a}}$ e $4^{\mathrm{a}}$ cadeiras; $5^{\circ}$ ano: $1^{\mathrm{a}}, 2^{\mathrm{a}}, 3^{\mathrm{a}}$ e $4^{\mathrm{a}}$ cadeiras), e encerrando com a última parte intitulada notas $\mathrm{e}$ correções.

O objetivo central do trabalho de Theodoro foi estudar a teoria das funções de variáveis reais sobre a noção de polinômio, utilizando a representação efetiva das funções

no ano seguinte foi nomeado professor de matemática da Escola Politécnica do Rio de Janeiro. Foi professor de Mecânica Racional.

${ }^{6}$ Francisco Bhering (1867 - 1924): Foi professor de Astronomia na Escola Politécnica do Rio de Janeiro.

${ }^{7}$ Augusto de Brito Belford Roxo (1878 -?): Foi diplomado em Engenharia Civil em 1900 pela Escola Politécnica do Rio de Janeiro e, posteriormente, professor de Estabilidade da mesma.

${ }^{8}$ Maurício Joppert da Silva (1890- 1985): Foi professor de Portos e livre docente de Cálculo Diferencial e Integral da Escola Politécnica do Rio de Janeiro.

RBHM, Vol. 19, n 38, p. 23-44, 2019 
somáveis de uma variável. Este resultado foi obtido por Lebesgue como consequência de um critério geral aplicado às integrais singulares das funções somáveis de uma variável real e, difere da demonstração dada por Theodoro por ser mais direta, bem como, a representação das funções somáveis de duas variáveis, um resultado obtido antes por Leonida Tonelli, que examinou os polinômios de Landau de duas variáveis. Consistiu assim, como escopo principal do trabalho de Theodoro examinar a representação das funções somáveis de duas variáveis por meio da integral dupla de Weierstrass.

Os resultados utilizados por Theodoro Augusto Ramos e que foram obtidos por Borel, Baire, Lebesgue, Tonelli e Weierstrass, datam do final do século XIX e início do século XX. O trabalho destes autores teve notável importância para o desenvolvimento das investigações matemáticas empreendidas pelo personagem em estudo, e corroboraram a contemporaneidade de sua pesquisa. No prefácio escrito por Theodoro, lê-se:

"A theoria das funcções de variaveis reaes que até o último decennio do seculo passado jazia em relativo esquecimento, desenvolveu-se de modo extraordinario após os trabalhos de E. Borel sobre a medida dos conjunctos e as teses de R. Baire e H. Lebesgue sobre as funcções discontinuas e a integração. Recentes estudos visam principalmente a simplificação da exposição geral da theoria. Constitui um dos objectivos deste modesto trabalho mostrar como se é naturalmente levado a basear a theoria das funcções de variaveis reaes sobre a simples noção de polynomio". (RAMOS, 1918, p.3)

Ao adentrar no trabalho produzido por Theodoro, nota-se que esse observou que em sua época os estudos estavam visando "principalmente a simplificação da teoria" (RAMOS, 1918, p. 3). Após uma explanação dos seus objetivos, o autor pontua também que:

As funcções limites que se obtem pela consideração exclusiva da equiconvergencia ou da convergencia de successões de polynomios, possuem propriedade bem características: são respectivamente as funcções de classe zero (funcções continuas) e as funcções de classe 1. As funcções de outras classes não poderão, portanto, ser representadas por successões de polynomios em todo o dominio em que são definidas. Faz-se mister, pois, excluir deste dominio certas porções onde se manifestam as singularidades que impedem a convergência das successões de polynomios. Entretanto, para que se possa tirar desta generalização consequencias uteis, necessário é que as porções excluídas tenham uma medida arbitrariamente pequena. Chega-se assim á noção de convergencia simples de polynomios. Aliás, mediante um estudo prévio das successões de funcções de Baire verifica-se effectivamente a possibilidade de edificar a theoria sobre esta noção. Convém observar que na definição de convergencia simples a única noção nova que intervem é a de conjuncto de medida nulla, noção esta que pode ser adquirida independentemente da theoria geral da medida. [...] Seguimos, entretanto, marcha differente. 
Consideramos as funcções como limites de successões de polynomios simplesmente convergentes, e baseando-nos nesta noção definimos a integral de uma funcção limitada. Esta definição é, pois diversa da de E. Borel e também da de F. Riesz. Afastamo-nos ainda deste ultimo auctor fazendo intervir no desenvolvimento da theoria considerações attinentes á theoria geral da medida. Assim procedemos pelos motivos que seguem. Primeiramente não achamos muito justa a critica de Riesz, pois adoptando os methodos de Borel extremamente simples se torna o estudo da theoria geral da medida. Em segundo lugar, acceitando o auxilio desta ultima theoria, conseguimos dar maior unidade á nossa exposição fazendo com que os resultados obtidos sejam consequencias logicas de uma unica proposição que denominamos theorema fundamental. Esta proposição foi demonstrada por Egoroff no tomo 152 dos Comptes-Rendus (1912); a sua demonstração differe, aliás, em alguns pontos da que apresentamos. [...] Em nosso trabalho estudamos primeiramente as funcções de uma variavel real; consideramos depois as funcções de duas variaveis reaes. Estudamos também a representação effectiva das funcções sommaveis de uma e de duas variaveis pela integral de Weierstrass. No caso em que a funcção é de uma variavel este resultado já foi obtido por Lebesgue como consequencia de um criterio geral applicavel ás integraes singulares das funcções sommaveis de uma variavel. Esta memória de H. Lebesgue foi publicada no tomo I, 3. ${ }^{a}$ série dos Annales de la Faculté dês Sciences de Toulouse. Conservamos, entretanto a nossa demonstração que differe da de Lebesgue, pois é directa. Quanto á representação effectiva das funcções sommaveis de 2 variaveis, L. Tonnelli fez um estudo relativo aos polynomios de Landau de 2 variaveis (vide a referencia que a este respeito faz M. Fréchet na pág. 226 do t.2, vol. 1, $2^{\circ}$ fasciculo da "Encyclopédie dês Sciences Mathématiques”, edição francesa). Propomo-nos a estudar a representação das funcções sommaveis de 2 variaveis pela integral dupla de Weierstrass". (RAMOS, 1918, p.3-6)

Desta forma, no estudo da tese em questão destacam-se implicações importantes.

Iniciando o texto, o prefácio da tese dedica-se a uma exposição geral do que será tratado pelo autor, e a introdução apresenta algumas definições que serão utilizadas no desenvolvimento da investigação. Dentre estas definições destaca-se a de conjunto de medida nula como sendo aquele cujos pontos podem ser encerrados em um número finito ou uma infinidade numerável de conjuntos elementares de medida total inferior a $\varepsilon$, $\varepsilon$ sendo arbitrariamente pequeno. O conjunto formado pela reunião de um número finito ou de uma infinidade numerável de conjuntos de medida nula também tem uma medida nula.

Observa que um conjunto numerável tem, evidentemente, uma medida nula e vê-se que o conjunto de medida nula pode ser denso em qualquer intervalo, pois é de tal natureza o conjunto dos números racionais.

Este conceito permite uma generalização devida a E. Borel e que foi utilizada no trabalho: Pode-se fazer uma extensão da definição de medida a certos conjuntos cuja medida 
não tenha sido definida. Assim, se um conjunto (E) contém todos os pontos de um conjunto mensurável $\left(\mathrm{E}_{1}\right)$, diremos que a medida de $(\mathrm{E})$ é superior ou igual à de $\left(\mathrm{E}_{1}\right)$, ainda que não tenhamos indicações sobre a natureza de $(\mathrm{E})$. Inversamente, se o conjunto mensurável $\left(\mathrm{E}_{1}\right)$ contém todos os pontos do conjunto $(\mathrm{E})$, diremos que a medida de $(\mathrm{E})$ é inferior ou igual à de $\left(E_{1}\right)$. A adoção destas definições generalizadas equivale a substituir um cálculo de igualdades por um cálculo de desigualdades. Esta generalização foi o ponto de partida de H. Lebesgue para a sua definição de medida dos conjuntos.

Outra definição apresentada por Theodoro e que foi utilizada no desenvolvimento de seu resultado principal é o de convergência uniforme. Theodoro a define:

"Supondo que no intervalo $(a, b)^{9}$ em que as funções $f_{n}(x)$ são definidas, $a$ função $r(x)$ seja limitada, a convergência de $\left[f_{n}(x)\right]$ para a função limite $f(x)$ é dita uniforme)." (RAMOS, 1918, p. 19)

A função $r(x)$ é dada como segue:

"Pelo critério de Cauchy sobre limites, conclui-se que sendo dados um valor $x_{1}$ do intervalo $(a, b)$, e um número positivo $\varepsilon$, a esses números corresponde um valor $r$ tal que se tenha:

$$
\left|f\left(x_{1}\right)-f_{n}\left(x_{1}\right)\right|<\varepsilon \text { para } n>r .
$$

Dado $\varepsilon$, este número $r$ no caso geral depende do valor de $x$ considerado; seja $r(x)$ o menor dos valores de $r$ que satisfazem a condição precedente quando $x$ e e são dados. A função $r(x)$ é finita em todo o intervalo $(a, b)$ mas pode não ser limitada para cada valor fixo de $\varepsilon$. Vê-se, assim, que se uma sucessão $\left[f_{n}(x)\right]$ é uniformemente convergente no intervalo $(a, b)$, a todo número positivo $\varepsilon$, por menor que seja, corresponde um número $r$ tal que se tenha em todo o intervalo $(a, b)$ :

$$
\left|f(x)-f_{n}(x)\right|<\varepsilon \text { para } n>r .
$$

Podem-se reunir as condições necessárias e suficientes para que $\left[f_{n}(x)\right]$ tenha um limite e tenda uniformemente para este limite, dizendo: $\left[f_{n}(x)\right]$ tem um limite para $n$ infinito e tende uniformemente para o seu limite no intervalo $(a, b)$, quando a cada número positivo $\varepsilon$ pode-se fazer corresponder um inteiro $r$ tal que se tenha:

$$
\left|f_{n}(x)-f_{m}(x)\right|<\varepsilon \text { para } n>r \text { e } m>r,
$$
qualquer que seja o ponto $x$ do intervalo $(a, b)$." (RAMOS, 1918, p. 18)

Na primeira parte da tese, intitulada As funcções de uma variável real, observam-se algumas definições importantes. A saber, a definição de continuidade de uma função em determinado intervalo dada por Theodoro:

Diremos que $f(x)$ é contínua no intervalo $(a, b)$ quando existe uma sucessão de polinômios $\left[P_{n}(x)\right]$ convergindo uniformemente para $f(x)$ em todos os

\footnotetext{
${ }^{9}$ ( , ): Notação para intervalo fechado. Utilizada em todos os casos que for descrita como advindas de Theodoro.
} 
pontos deste intervalo. Nestas condições, sendo dado o número positivo $\varepsilon$, por menor que seja, pode-se achar um número $r$ tal que se tenha $\left|f(x)-P_{n}(x)\right|<\varepsilon$, para todos os valores de $n$ superiores a $r$, e para todos os pontos de $(a, b)$. Diremos que $f(x)$ é contínua num ponto $x_{0}$ no sentido restrito e no qual $f(x)$ é contínua. (RAMOS, 1918, p.21)

Define-se continuidade à direita e à esquerda: $f(x)$ sendo contínua no intervalo $(a, b)$, tem-se na sua extremidade $a,\left|f(a)-P_{n}(a)\right|<\varepsilon$, para $n>r$ mas não se pode dizer que $f(x)$ é contínua no ponto $a$, por não ser este ponto interior no sentido restrito $a$ nenhum intervalo em que $f(x)$ é contínua; diremos então que $f(x)$ no ponto $a$ é contínua à direita. Do mesmo modo $f(x)$ é contínua à esquerda, no ponto $b$.

Theodoro utiliza-se desta definição e de duas propriedades que seguem, para apresentar a definição de Cauchy de função contínua. Destaca-se que a definição de continuidade de Cauchy é a propriedade II de Theodoro apresentada abaixo.

"Propriedade I: Se $f(x)$ é contínua em $(a, b)$, dado um número positivo $\epsilon$, por menor que seja, pode-se achar um número positivo $\delta$, tal que dividindo $(a, b)$ em intervalos de comprimento não superior $a \delta$, se tenha, $\left|f\left(x_{1}\right)-f\left(x_{0}\right)\right|<\epsilon, x_{1}$ e $x_{0}$ designando dois pontos quaisquer de um desses pequenos intervalos.

Propriedade II: Fazendo $x_{1}-x_{0}=h$, vem $\left|f\left(x_{0}+h\right)-f\left(x_{0}\right)\right|<\epsilon$ para $|h|<a$, e podemos enunciar: Se $f(x)$ é contínua no intervalo $(a, b)$, $e$ se o ponto $x_{0}$ é interior a $(a, b)$ no sentido restrito, a cada valor da quantidade positiva $\epsilon$, por menor que seja, corresponde um número positivo a tal que se tenha $\left|f\left(x_{0}+h\right)-f\left(x_{0}\right)\right|<\epsilon$ para $|h|<a$. Nas extremidades a e $b$, tem-se respectivamente $|f(a+h)-f(a)|<\epsilon \quad e$ $|f(b-h)-f(b)|<\epsilon$ para $|h|<a, \quad h$ tomando somente valores positivos". (RAMOS, 1918, p.22)

Após enunciar as duas propriedades, Theodoro faz a seguinte observação:

"Cauchy definiu a continuidade de uma função por esta segunda propriedade: portanto se uma função é contínua pela definição que demos é também contínua no sentido de Cauchy. Como vimos a segunda propriedade é apenas uma consequência da primeira, inversamente Cantor e Heine demonstraram que as funções contínuas no sentido de Cauchy possuem a primeira propriedade que recebeu a denominação de propriedade da continuidade uniforme. A proposição de Cantor e Heine pode ser facilmente demonstrada com o auxílio do teorema de Borel Lebesgue. (RAMOS, 1918, p. 23)

Do teorema que segue, pode-se concluir que a função limite de uma sucessão ou de uma série uniformemente convergente de funções contínuas, também é contínua. 
Teorema de Weierstrass: Seja $f(x)$ uma função contínua no sentido de Cauchy, definida no intervalo $(a, b)$. Dado o número positivo $\varepsilon$, por menor que seja, pode-se achar um polinômio $P(x)$ tal que se tenha em todo o intervalo $(a, b),|f(x)-P(x)|<\varepsilon$. Segundo o autor:

"A convergencia uniforme de uma successão de funç̧ões continuas é evidentemente uma condição sufficiente para que a funcção limite seja contínua; não é, porém, uma condição necessaria, isto é, há successões de funcções contínuas que não sendo uniformemente convergentes tendem, entretanto, para funç̧ões continuas. Arzela demonstrou que a condição necessaria e sufficiente para que a funcção limite de uma successão de funcções continuas seja continua é que a convergencia desta successão seja quasi-uniforme”. (RAMOS, 1918, p.28)

A definição de convergência quase uniforme para Theodoro:

Definição (Convergência quase uniforme): Uma sucessão converge quase uniformemente em $(a, b)$, quando: $1^{\circ}$ - a sucessão converge em $(a, b) ; 2^{\circ}$ sendo dados epositivo arbitrariamente pequeno e um número $N$, por maior que seja, pode-se achar um número finito $N^{\prime} \geq N$ tal que para cada valor de $x$ de $(a, b)$ existe um número inteiro $n_{x}$ compreendido entre $N$ e $N$ ', para o qual $\left|f(x)-P_{n_{x}}(x)\right|<\varepsilon$. (RAMOS, 1918, p.28)

Como já apresentado acima, importante para entender o trabalho de Theodoro é a compreensão acerca de sua definição do conceito de continuidade. O autor esclarece e demonstra que, se uma função é contínua pela definição dada por ele, é também contínua na definição de Cauchy.

Na página 38 de sua tese, Theodoro faz a seguinte observação:

"Somos assim levados a estudar as funç̧ões discontinuas limites de successões convergentes de polynomios. Estas funcções foram designadas por $R$. Baire pelo nome de funcções de classe 1, ficando reservada ás funcções limites de successões uniformemente convergentes de polynomios, isto é ás funcções contínuas, a denominação de funç̧ões de classe zero". (RAMOS, 1918, p. 38)

Definição: Diz-se que um conjuncto $(E)$ é denso em um intervalo $(a, b)$ quando em um intervalo qualquer arbitrariamente pequeno contido em $(a, b)$ existem sempre pontos de (E).

No tocante à função pontualmente descontínua, Theodoro aponta:

"Diz-se que uma funç̧ão $f(x)$ é pontualmente descontínua no intervalo $(a, b)$ quando o conjunto dos seus pontos de descontinuidade é não-denso em $(a, b)$. Nestas condições em todo intervallo $(\alpha, \beta)$ interior (no sentido 
restricto) a ( $a, b)$ existem pontos onde $f(x)$ é contínua, isto é o conjuncto dos pontos de continuidade de $f(x)$ é denso em $(a, b)$ ". (RAMOS, 1918, p. 39)

Após a observação acima, que será mais bem explorada ao longo do texto, Theodoro demonstra o seguinte teorema: "Seja $\left[P_{n}(x)\right]$ uma successão de polynomios convergindo para a função limite $f(x)$ no intervalo $(a, b)$. Vamos mostrar que $f(x)$ é pontualmente descontínua em $(a, b)$ " (RAMOS, 1918, p. 39). Ou seja, que o conjunto de pontos de descontinuidades não é denso.

Uma notação: Para Theodoro conjunto perfeito é aquele que coincide com o seu derivado $^{10}$, ou seja, todos seus pontos são pontos de acumulação, pois não possui ponto isolado.

Então: a condição necessária e suficiente para que uma função seja de classe zero ou de classe 1 é que $f(x)$ seja pontualmente descontínua relativamente a todo conjunto perfeito.

Assim, a condição necessária e suficiente para que uma função seja de classe zero ou de classe 1 é que $f(x)$ seja pontualmente descontínua relativamente a todo conjunto perfeito.

As funções derivadas que em geral são de classe 1 possuem a seguinte propriedade que erradamente era atribuída somente às funções contínuas: uma função derivada não pode passar de um valor $A$ a outro $B$ sem passar por todos os valores intermediários.

São estes resultados de destaque que compõe a primeira parte da tese de Theodoro.

Passando a segunda parte da tese, ao tratar da teoria das funções somáveis, Theodoro apoia-se nos conceitos e teorias discutidos por René Baire em sua tese de doutoramento em Ciências Matemáticas apresentada a La Faculté des Sciences de Paris e, datada do ano de 1899. A título de curiosidade, a banca examinadora dessa tese foi constituída por MM. Darboux (presidente), Appell e Picard (examinadores).

Na página 44 da tese de Theodoro lê-se:

"R. Baire propoz uma classificação das funcções de variaveis reaes. As funcções continuas pertencem á classe zero e as funcções discontinuas limites de funcções continuas á classe 1. De um modo geral, são denominadas funcções de classe $n$ as funç̧ões limites de funcções de classe $n-1$ que não pertencem a esta classe ou a classe menor". (RAMOS, p.44, 1918)

Neste sentido, buscando os trabalhos de R. Baire, no capítulo III de sua tese, intitulado Fonctions discontinues développables em séries multiples de fonctions continues, no primeiro item relativo à definição destas funções, Baire (1899, p.68-69) escreve:

\footnotetext{
${ }^{10}$ Um conjunto $(E)$ pode ter um número finito ou uma infinidade de pontos de acumulação que constituem um outro conjuncto $\left(E^{\prime}\right)$ denominado conjuncto derivado de $(E)$.
}

RBHM, Vol. 19, n 38, p. 23-44, 2019 
"62. Eu me proponho definir e estudar neste capítulo, certas classes de funções descontínuas, que pode se dizer relacionar, em certo sentido, as funções contínuas. Tomarei como ponto de partida a noção de função limite de uma sequência de funções. Acabamos de ver, no capítulo anterior, existem funções descontínuas de uma variável real que podem ser obtidas como limites de funções contínuas, e determinou-se a condição necessária e suficiente para que uma função tenha esta propriedade. Eu concordo em dizer que as funções contínuas formam a classe $0, e$ as funções descontínuas limites de funções contínuas formam a classe 1. De acordo com isso, as funções da primeira classe são funções que são descontínuas representáveis por séries convergentes de funções contínuas e, consequentemente, como já demonstrado, por séries convergentes de polinômios.

63. Suponha agora que temos uma sequência de funções pertencentes às classes 0 ou 1, e com uma função limite de não pertencer a qualquer uma destas duas classes. Diria que esta função limite é uma função da segunda classe, e o conjunto de todas as funções que podem ser obtidos desta forma irão formar classe 2. É visto a partir deste que uma função de classe 2 pode ser expandida em uma série, convergente para cada valor de $x$, e cujos termos são funções de classe 1, através da substituição de cada termo pelo conjunto de polinômios que representa, é reconhecido que uma função de classe 2 pode ser representada por uma série dupla cujos termos são polinômios.

64. Assim como nós definimos as funções da classe 1 e 2, podemos definir as funções das classes 3, 4, ... n, ... Uma função é dita ser da classe $n$, se ela é o limite de uma sequência de funções pertencentes as classes 0,1, 2, ..., $n-1$, e se ela própria não pertence a uma dessas classes. Tal função, se houver, será representado por uma série de ordem $n$, cujos termos são polinômios: $\sum_{a_{1}} \sum_{a_{2}} \ldots \sum_{a_{n}} P_{a_{1} a_{2} \ldots a_{n}}(x)^{\prime \prime .}{ }^{11}$ (BAIRE, 1889, p. 68-69)

\footnotetext{
${ }^{11}$ 62. Je me propose de definir et d'étudier dans ce chapitre, certaines catégories de fonctions discontinues, dont on peut dire qu'elles se rattachent, en un certain sens, aux fonctions continues. Je prendrai pour point de départ la notion de fonction limite d'une suite de fonctions. Nous venons de voir, dans le chapitre précédent, qu'il y a des fonctions discontinues d'une variable réelle qu'on peut obtenir comme limites de fonctions continues, et nous avons déterminé la condition nécessaire et suffisante pour qu'une fonction possède cette propriété. Je conviendrai de dire que les fonctions continues forment la classe 0 , et que les fonctions discontinues limites de fonctions continues forment la classe 1. D'après cela, les fonctions de la première classe sont les fonctions discontinues qui sont représentables par des séries convergentes de fonctions continues, et par suite, comme nous l'avons montré, par des séries convergentes de polynômes. 63. Supposons maintenant qu'on ait une suite de fonctions appartenant aux classes 0 ou 1, et possédant une fonction limite n'appartenant à aucune de ces deux classes. Je dirai que cette fonction limite est une fonction de la second classe, et l'ensemble de toutes les fonctions qu'on peut obtenir de cette manière formera la classe 2 . On voit d'après cela qu'une fonction de la classe 2 est développable en une série, convergente pour chaque valeur de $x$, et dont tous les termes sont des fonctions de classe 1 ; en remplaçant chacun de ces termes par la série de polynômes qui le représente, on reconnâit qu'une fonction de classe 2 peut être représentée par une série double dont les termes sont des polynômes. [...] 64. Dê même que nous avons défini les fonctions des classes 1 et 2, nous pourrons définir les fonctions de classes $3,4, \ldots \mathrm{n}, \ldots$ Une fonction sera dite de classe $n$, si elle est la limite d'une suite de fonctions appartenant aux classes $0,1,2, \ldots, \mathrm{n}-1$, et si elle n'appartient pas elle-même à l'une de ces classes.
} 
O que coincide com a definição atual acerca da classificação de Baire.

Um resultado importante neste sentido é a demonstração do teorema fundamental relativo às sucessões simplesmente convergentes de funcções de Baire. Para um melhor entendimento, segue a definição:

"Diz-se que $\left[f_{n}(x)\right]$ converge simplesmente para a funç̧ão $f(x)$ quando $f_{n}(x)$ tende para $f(x)$ em todos os pontos de $(a, b)$ excepto talvez nos pontos de um conjuncto de medida nulla. É evidente que a convergência no sentido commum é um caso particular da convergência simples ${ }^{12 " .}$. (RAMOS, 1918, p. 45)

Teorema:

"Seja $\left[f_{n}(x)\right]$ uma sucessão de funções de Baire convergindo simplesmente para uma função limite $f(x)$ no intervalo $(a, b)$. Sendo dado o número positivo $\sigma$, por menor que seja, existe no intervalo $(a, b) \mathrm{um}$ conjunto de medida maior que $b-a-\sigma$ no qual $\left[f_{n}(x)\right]$ converge uniformemente". (RAMOS, 1918, p.45)

Outros teoremas e resultados que seguem podem ser tomados como relevantes para o desenvolvimento da tese de Theodoro.

O $2^{\circ}$ Teorema Básico:

"Seja $f(x)$ uma função de Baire definida no intervalo $(a, b)$ e tal que dado o número positivo arbitrariamente pequeno $\sigma_{1}$, exista uma sucessão de funções tendendo uniformemente para $f(x)$ em um conjunto de medida superior $a b-a-\sigma_{1}$. Nestas condições é possível achar uma sucessão de funções $\left[f_{n}(x)\right]$ que tende simplesmente para $f(x)$ em $(a, b)$ ”. (RAMOS, 1918, p.48)

Observa-se que este teorema intitulado por Theodoro como $2^{\circ}$ Teorema Básico é uma recíproca para o primeiro acima (não identificado como $1^{\circ}$ Teorema Básico pelo autor, mas simplesmente nomeado teorema).

O $3^{\circ}$ Teorema Básico:

"Seja $\left[f_{n}(x)\right]$ uma sucessão de funções de Baire convergindo simplesmente para $f(x)$ no intervalo $(a, b)$. Se cada função $f_{n}(x)$ for limite

Une telle fonction, s'il en existe, pourra se représenter par une série d'ordre $n$, dont les termes seront des polynômes: $\sum_{a_{1}} \sum_{a_{2}} \ldots \sum_{a_{n}} P_{a_{1} a_{2} \ldots a_{n}}(x)$.

${ }^{12}$ Consideremos uma sucessão de funções de uma variável real definidas no intervallo $(a, b)$ e que representaremos pelo símbolo $\left[f_{n}(x)\right]$ tenda para todos os valores $x$ de $(a, b)$ para uma funcção $f(x)$ quando $\mathrm{n}$ cresce indefinidamente; tem-se então: $\lim _{n=\infty} f_{n}(x)=f(x)$, e a successão é convergente no intervallo $(a, b)$.

RBHM, Vol. 19, n 38, p. 23-44, 2019 
de uma sucessão de polinômios simplesmente convergente $f(x)$ também será limite de uma sucessão simplesmente convergente de polinômios". (RAMOS, 1918, p.49)

Destes teoremas, vem:

A conclusão imediata do $3^{\circ}$ Teorema Básico: Toda função pertencente à classificação de Baire pode ser considerada como limite de uma sucessão simplesmente convergente de polinômios. $\mathrm{E}$, se a sucessão de funções somáveis $\left[f_{n}(x)\right]$ tende simplesmente no intervalo $(a, b)$ para uma função limite $f(x)$, a função $f(x)$ também é somável.

A conclusão: Todas as funções limitadas pertencentes à classificação de Baire são somáveis.

Com auxílio dos três teoremas acima, o autor irá adentrar a integração das funções somáveis, além de abordar o estudo das funções limitadas pertencentes à classificação de Baire desenvolvendo a teoria de integração de tais funções.

Define: "Seja $f(x)$ uma funcção, limitada e definida no intervallo $(a, b)$. Se $f(x)$ é limite de uma successão de polynomios $\left[P_{n}(x)\right]$ simplesmente convergente, diz-se que $f(x)$ é uma funcção somável " (RAMOS, 1918, p.51).

"A função integral de uma função somável $f(x)$ é o limite das integrais dos termos de uma sucessão de polinômios $\left[P_{n}(x)\right]$ que converge simplesmente para $f(x)$ " (RAMOS, 1918, p.53).

Assim, passa ao teorema que constituiu seu próximo objetivo:

“Seja $\left[f_{n}(x)\right]$ uma sucessão de funções somáveis limitadas em conjunto, $e$ tendendo simplesmente para a função somável $f(x)$ no intervalo $(a, b) . A$ integral de $f_{n}(x)$ tende para a integral de $f(x)$ ". (RAMOS, 1918, p. 56)

Theodoro utilizará deste resultado nos encaminhamentos do seu teorema principal discutido à frente.

Segundo Theodoro é importante o teorema: "As integrais indefinidas de uma função derivada limitada são as suas funções primitivas” (RAMOS, 1918, p.58).

Na página 60, o autor fala sobre a generalização do conceito de integral e do adotado por ele em sua tese:

"Comparando os resultados precedentes com os trabalhos de Borel e Lebesgue relativos á generalização de integral, vemos que suppondo as funcções limitadas, a definição que apresentamos possue as mesmas propriedades que as definições d'aquelles autores. A equivalência entre estas definições é aliás, posta em evidencia por 2 theoremas dos quaes o primeiro foi demonstrado por H. Lebesgue (vide Leçons sur les séries trigonométriques, pg.14) e assim se enuncia: "Si na successão de funções sommaveis no sentido de Lebesgue converge simplesmente para a função sommavel $f(x)$, a successão das integraes converge para a integral de 
$f(x)$ ". Quanto ao segundo theorema que foi demonstrado por F. Riesz no t. 148 dos Comptes-Rendus, o seu enunciado é o seguinte: "Si uma successão de funcções converge asymptoticamente para uma funcção limite, desta successão póde-se extrahir uma outra que converge simplesmente para a mesma funcção limite. A noção de integral generalizada é capital no estudo das funções derivadas e na pesquiza das funcções primitivas. H. Lebesgue obteve os principais resultados sobre o assumpto",". (RAMOS, 1918, p.60)

Esses resultados permitiram ao autor considerar as funções como limites de sucessões de polinômios simplesmente convergentes e, baseando nesta noção, definir a integral de uma função limitada. Para esta definição, utilizou das propriedades da integral de Lebesgue e da integral de Borel, com o intuito de construir a parte preliminar da teoria da integração das funções limitadas sem a intervenção da teoria geral da medida.

A seguir Theodoro passou ao estudo da representação efetiva das funções somáveis.

$\mathrm{O}$ autor explica que se encontra no livro de E. Borel "Leçons sur les fonctions de variables réelles" uma demonstração do teorema de Weierstrass relativo às funções contínuas. Nos fala que como consequência desta demonstração, devida a Weierstrass nos seus pontos essenciais, consegue-se representar uma função contínua qualquer pela expressão:

$$
\psi(x, k)=\frac{1}{k \sqrt{\pi}} \int_{-\infty}^{+\infty} f(u) e^{-\left(\frac{u-x}{k}\right)^{2}}
$$

Continua dizendo que:

"Lendo o estudo que fez Lebesgue nas suas "Leçons sur les séries trigonométriques" sobre a representação de uma funcção sommavel pelas sommas de Féjer, tivemos a idéa de realizar identica generalização para a integral $\psi(x, k)$, utilizando parte dos raciocinios alli adoptados, generalização esta que aqui apresentamos. Este resultado já foi, entretanto obtido por H. Lebesgue como consequencia de um criterio geral applicavel ás integrais singulares das funcções sommaveis de uma variavel. A memória de H. Lebesgue sobre este assumpto foi publicada nos Annales de la Faculté des Sciences de Toulouse, $3^{a}$ série, toma I, que acabamos que receber da Europa. Conservamos a nossa demonstração que differe da de Lebesgue por ser directa. A demonstração de Lebesgue relativas ás sommas de Féjer que se encontra nas "Leçons sur les séries trigonométriques" está visivelmente errada. M. Fréchet no seu artigo de Encyclopédie des Sciences Mathématiques (t. III, $1^{\circ}$ vol., $2^{\circ}$ fasciculo, edição francesa, 1912), diz que a demonstração primitiva de H. Lebesgue publicada na revista alemã Math. Ann. 61, (1905), que não conhecemos, acha-se isenta de erros. Na sua memoria já citada nos Annales de la F. des $S$. de Toulouse, $H$. Lebesgue rectifica o seu engano e mostra que a referida 
proposição pode ser obtida como consequencia do criterio geral relativo ás integraes singulares". (RAMOS, 1918, p. 64)

Percebe-se por esta citação de Theodoro o resultado central de sua tese. Mesmo com a demonstração de Lebesgue, o mesmo explica o motivo de manter sua demonstração.

Assim, Theodoro considerará uma função somável $f(x)$ definida no intervalo $(a, b)$. Explica que fora deste intervalo define-se $f(x)$ do seguinte modo:

Em $(-\infty, a), f(x)=f(a)$ e em $(b,+\infty), f(x)=f(b)$. Esclarece ter assim definido uma função somável em $(a, b)$ e limitada em $(-\infty,+\infty)$.

$\mathrm{O}$ autor passa ao resultado:

Teorema: A expressão

$$
\psi(x, k)=\frac{1}{k \sqrt{\pi}} \int_{-\infty}^{+\infty} f(u) e^{-\left(\frac{u-x}{k}\right)^{2}} d u, k>0
$$

tende para $f(x)$ quando $k$ tende para zero, em todos os pontos de $(a, b)$, exceto talvez nos pontos de um conjunto de medida nula.

O que resulta a possibilidade de conseguir representar uma função continua qualquer pela expressão acima. importantes:

Para a demonstração deste teorema Theodoro utiliza de alguns resultados

a. Lema: O conjunto dos pontos nos quais

$$
\Phi^{\prime}(0)=\lim _{t=0} \frac{1}{t} \int_{0}^{t}|\varnothing(t)| d t=0
$$

tem como complementar em relação ao intervalo $(a, b)$ um conjunto de medida nula.

b. Resultado 1: Theodoro começa a estudar a expressão

$$
\psi(x, k)=\frac{1}{k \sqrt{\pi}} \int_{-\infty}^{+\infty} f(u) e^{-\left(\frac{u-x}{k}\right)^{2}} d u
$$

e considera $M$ o limite superior de $|f(x)|$ em $(-\infty,+\infty)$. Para este estudo, separa $\psi(x, k)$ em três partes: $\quad \psi(x, k)=\frac{1}{k \sqrt{\pi}} \int_{-\infty}^{x-h}+\frac{1}{k \sqrt{\pi}} \int_{x-h}^{x+h}+\frac{1}{k \sqrt{\pi}} \int_{x+h}^{+\infty}$.

Ou seja, fazendo $u=x+k t$, tem-se:

$$
\sqrt{\pi} \psi(x, h)=\int_{-\infty}^{-\frac{h}{k}} f(x+k t) e^{-t^{2}} d t+\int_{-\frac{h}{k}}^{+\frac{h}{k}} f(x+k t) e^{-t^{2}} d t+\int_{+\frac{h}{k}}^{+\infty} f(x+k t) e^{-t^{2}} d t
$$

Estudando as integrais separadamente e utilizando de algumas operações e do lema (a), obtém os resultados:

Considere a função abaixo: 


$$
\begin{gathered}
g(x, k)=\int_{-\frac{h}{k}}^{+\frac{h}{k}} f(x+k t) e^{-t^{2}} d t \\
|g(x, k)-f(x)|<\epsilon+\frac{2 \epsilon}{\sqrt{\pi}}|f(x)|<\epsilon+\frac{2 \epsilon}{\sqrt{\pi}} M\left(^{*}\right) \\
|\psi(x, k)-g(x, k)|<2 M \epsilon(* *)
\end{gathered}
$$

Por $(*)$ e $(* *)$, conclui-se que:

$$
|\psi(x, k)-f(x)|<\epsilon+4 M \epsilon
$$

Logo, o conjunto dos pontos em que $\Phi^{\prime}(0)=0$ tem como complementar em relação a $(a, b)$ um conjunto de medida nula; concluímos assim que

$$
\psi(x, k)=\frac{1}{k \sqrt{\pi}} \int_{-\infty}^{+\infty} f(u) e^{-\left(\frac{u-x}{k}\right)^{2}} d u
$$

tende para a função somável $f(x)$, quando $k$ tende para zero, em todos os pontos de $(a, b)$, exceto talvez nos pontos de um conjunto de medida nula.

c. Resultado 2:

Da conclusão acima,

"Deduz-se a possibilidade de representar no intervallo $(a, b)$ a funcção contínua $f(x)$ por uma successão de polynomios uniformemente convergente. Com effeito, em um intervalo finito qualquer $(a, b), \psi(\mathrm{x}, \mathrm{k}) e ́$ desenvolvível em serie inteira segundo as potências crescentes de $x$ (Vide Borel "Leçons sur les fonctions de variables réelles", pg. 54); tomando um numero sufficiente de termos desta serie, consegue-se representar em $(a, b), \psi(\mathrm{x}, \mathrm{k})$ e portanto $f(x)$, com a approximação que se quizer por um polynomio”. (RAMOS, 1918, p. $72-73$ )

Adentrando à terceira parte da tese onde são tratadas as notas 1 e 2 estudadas pelo autor, tem-se:

Na nota 1 intitulada Nota sobre uma fórmula de interpolação, o autor faz algumas considerações e dedica-se principalmente a demonstrar que se a função contínua $f(x)$ em $(0,1)$, satisfaz a condição de Lipschitz, este erro é um infinitésimo de ordem de $\frac{1}{\sqrt{\mathrm{m}}}$ pelo menos. Não há destaque de outros resultados.

Quanto à nota 2 intitulada Nota sobre a approximação das funcções duas vezes deriváveis, destaca-se o polinômio procurado:

De acordo com Theodoro, S. Bernstein (Note sur les séries normales, t. II, Leçons sur les principes de l'Analyse de R. D'Adhémar) partindo da fórmula:

$$
\text { (1) } f(x)=\frac{1}{2} \int_{0}^{1}|x-a| f^{\prime \prime}(a) d a+A+B x
$$


em que A e B são duas constantes, obteve para a representação de $f(x)$ um desenvolvimento em série normal, isto é, em série da forma:

$$
f(x)=\sum_{q=0}^{\infty} \sum_{p=0}^{\infty} A_{p, q} x^{p}\left(1-x^{2}\right)^{q}
$$

E ele se propõe a calcular efetivamente um polinômio que represente $f(x)$ com uma aproximação dada a priori.

Theodoro efetua alguns cálculos (cita M. Potron e seu texto "Sur une formule générale d'interpolation" - Bulletin de la Societé Mathématique de France, 1906) e chega ao polinômio procurado:

$$
P(x)=A+B x+\sum_{k=0}^{k=2 \delta m^{2}} C_{k} x^{k} .
$$
Funcional.

Sabe-se que polinômios dessa forma merecem destaques nos estudos de Análise

Na quarta e última parte da tese, o autor realizou algumas considerações sobre as funções de duas variáveis reais passando a representação efetiva das funções somáveis de duas variáveis reais pela integral dupla de Weierstrass. Nosso personagem fez a seguinte explanação:

"Vamos tratar directamente o caso geral das funç̧ões pertencentes a uma classe qualquer de Baire. Para as funç̧ões contínuas e funcções de classe 1 e 2 variaveis, grande parte dos resultados relativos ao caso de uma variável pode ser facilmente generalizada. A demonstração do theorema de Weierstrass pelo methodo de Lebesgue, para o caso de 2 variaveis, encontra-se, por exemplo, na pg. 63 do livro de Borel "Leçons sur les fonctions de variables réelles". (RAMOS, 1918, p.84)

Consideremos uma sucessão de funções da classificação de Baire $\left[f_{n}\left(x_{1}, x_{2}\right)\right]$ definidas no domínio mensurável D. Diz-se que $\left[f_{n}\left(x_{1}, x_{2}\right)\right]$ converge simplesmente para a função $f\left(x_{1}, x_{2}\right)$ quando $\left[f_{n}\left(x_{1}, x_{2}\right)\right]$ tende para $f\left(x_{1}, x_{2}\right)$ em todos os pontos de $D$, exceto talvez nos pontos de um conjunto de medida nula.

Destacam-se os resultados: $\mathrm{O}$ teorema fundamental, $2^{\circ}$ e $3^{\circ}$ teorema básico para o caso de duas variáveis reais.

a. Teorema fundamental - Seja $\left[f_{n}\left(x_{1}, x_{2}\right)\right]$ uma sucessão de funções de Baire convergindo simplesmente para uma função limite $f\left(x_{1}, x_{2}\right)$ no domínio mensurável $D$. Dado o número positivo $\sigma$, por menor que seja, pode-se achar no domínio $D$ um outro domínio cuja medida difere da de $D$ de menos de $\sigma$, e no qual $\left[f_{n}\left(x_{1}, x_{2}\right)\right]$ converge uniformemente.

$b$. Teorema II: Seja $f\left(x_{1}, x_{2}\right)$ uma função de Baire definida no domínio mensurável $D$ e tal que dado o número positivo arbitrariamente pequeno $\sigma$, exista uma sucessão de funções tendendo uniformemente para $f\left(x_{1}, x_{2}\right)$ em um domínio de medida superior a med $D-\sigma$. Nestas condições é possível achar uma sucessão de funções $\left[f_{n}\left(x_{1}, x_{2}\right)\right]$ que tende simplesmente para $f\left(x_{1}, x_{2}\right)$ no domínio $D$. 
c. Teorema III: Seja $\left[f_{n}\left(x_{1}, x_{2}\right)\right]$ uma sucessão de funções convergindo simplesmente para $f\left(x_{1}, x_{2}\right)$ no domínio mensurável $D$. Se cada função $f_{n}\left(x_{1}, x_{2}\right)$ for limite de uma sucessão de polinômios simplesmente convergente, $f\left(x_{1}, x_{2}\right)$ também será limite de uma sucessão simplesmente convergente de polinômios.

Theodoro passou ao tratamento das funções somáveis e define:

Definição (função somável de 2 variáveis): Seja $f\left(x_{1}, x_{2}\right)$ uma função limitada e definida no domínio mensurável $D$. Se $f\left(x_{1}, x_{2}\right)$ é limite de uma sucessão de polinômios $\left[P_{n}\left(x_{1}, x_{2}\right)\right]$ simplesmente convergente, diz-se que $f\left(x_{1}, x_{2}\right)$ é uma função somável.

E apresentou o resultado:

Teorema: Se a sucessão de funções somáveis tende simplesmente para uma função limite $f\left(x_{1}, x_{2}\right)$ no domínio mensurável $D, f\left(x_{1}, x_{2}\right)$ também é somável em D. (RAMOS, 1918, p.85)

Observa-se que este teorema é análogo ao já mencionado. Aqui Teodoro usa a ideia de medida, anteriormente ele não usa de maneira proposital. Ele faz para funções de duas variáveis aquilo que havia feito para funções de uma variável, mas só que usando a noção de medida.

Merece destaque o estudo realizado pelo autor relativo à representação das funções somáveis de 2 variáveis pela integral dupla de Weierstrass:

Seja $f\left(x_{1}, x_{2}\right)$ uma função somável definida no domínio mensurável $D$. Fora de $D$ definiremos $f\left(x_{1}, x_{2}\right)$ pondo $f\left(x_{1}, x_{2}\right)=0$.

A integral de Weierstrass é a seguinte:

$$
\psi\left(x_{1}, x_{2}, k\right)=\frac{1}{k^{2} \pi} \iint_{P} f\left(u_{1}, u_{2}\right) e^{-\left(\frac{u_{1}-x_{1}}{k}\right)^{2}-\left(\frac{u_{2}-x_{2}}{k}\right)^{2}} d u_{1} d u_{2},
$$

em que $k$ é positivo e o domínio de integração sendo todo o plano.

Será considerada a função:

$\phi\left(t_{1}, t_{2}\right)=f\left(x_{1}+t_{1}, x_{2}+t_{2}\right)+f\left(x_{1}-t_{1}, x_{2}+t_{2}\right)+f\left(x_{1}+t_{1}, x_{2}-t_{2}\right)$

$$
+f\left(x_{1}-t_{1}, x_{2}-t_{2}\right)-4 f\left(x_{1}, x_{2}\right)
$$

Para uma melhor compreensão da demonstração deste teorema dada por Theodoro, destacam-se os seguintes resultados:

a. Resultado 1: Lema: A relação

$$
\lim _{t=0} \frac{1}{t^{2}} \int_{0}^{t} \int_{0}^{t}\left|\phi\left(t_{1}, t_{2}\right)\right| d t_{1} d t_{2}=0
$$

é verificada em todos os pontos do domínio mensurável $\mathrm{D}$, excetuando talvez os pontos de um conjunto de medida nula.

b. Resultado 2: Estudo da integral $\psi\left(x_{1}, x_{2}, k\right)$.

Por uma escolha conveniente da origem, pode-se sempre supor o domínio mensurável $D$ interior a certo quadrado, que por sua vez é interior ao quadrado $\Delta$ de vértices 
$(0,0),(0, a),(a, 0),(a, a)$. Nestas condições qualquer que seja o ponto $D$, a sua distância a um ponto qualquer do contorno de $\Delta$ é superior a um número positivo fixo $\delta$.

Consideremos um ponto $M\left(x_{1}, x_{2}\right)$ qualquer do domínio $D$ e dividamos o quadrado $\Delta$ em 5 partes do seguinte modo: $\Delta_{1}$ é o quadrado de centro $M\left(x_{1}, x_{2}\right)$ e de lados paralelos aos de $\Delta$ e de comprimento $2 a<2 \delta$, quanto às partes $\Delta_{2} ; \Delta_{3} ; \Delta_{4} ; \Delta_{5}$; elas são obtidas mediante a divisão da parte de $\Delta$ que não pertence a $\Delta_{1}$, por 2 paralelas aos eixos coordenados traçadas pelo ponto $M$.

Temos, então:

$$
\iint_{P}=\iint_{\Delta_{1}}+\iint_{\Delta_{2}}+\iint_{\Delta_{3}}+\iint_{\Delta_{4}}+\iint_{\Delta_{5}}+\iint_{P_{1}}
$$

em que a última integral é referida a região $P_{1}$ do plano exterior ao quadrado $\Delta$.

Consideremos a integral:

$$
\frac{1}{k^{2} \pi} \iint_{\Delta_{2}} f\left(u_{1}, u_{2}\right) e^{-\left(\frac{u_{1}-x_{1}}{k}\right)^{2}-\left(\frac{u_{2}-x_{2}}{k}\right)^{2}} d u_{1} d u_{2} .
$$

$\mathrm{O}$ autor efetua alguns cálculos e conclui que $\frac{1}{k^{2} \pi} \iint_{\Delta_{2}}$ tende para zero com $k$, e isto qualquer que seja o ponto $M\left(x_{1}, x_{2}\right)$ de $D$.

Operando com as integrais $\iint_{\Delta_{3}}, \iint_{\Delta_{4}}, \iint_{\Delta_{5}}$ de modo análogo ao precedente, concluise que qualquer que seja o ponto $M\left(x_{1}, x_{2}\right)$ do domínio $\mathrm{D}$, as integrais

tendem para zero com $k$.

$$
\iint_{\Delta_{i}}(i=2,3,4,5,)
$$

A integral $\frac{1}{k^{2} \pi} \iint_{P_{1}}$ é evidentemente nula, pois em $P_{1}, f\left(x_{1}, x_{2}\right)=0$. Aliás, é fácil ver que se em $P_{1}, f\left(x_{1}, x_{2}\right)$ não fosse nula, mas tal que $\left|f\left(x_{1}, x_{2}\right)\right| \leq M, \frac{1}{k^{2} \pi} \iint_{\mathrm{P}_{1}}$ tenderia para zero com $\mathrm{k}$.

Acrescenta que resta então estudar a integral:

$$
g\left(x_{1}, x_{2}, k\right)=\frac{1}{k^{2} \pi} \iint_{\Delta_{1}} f\left(u_{1}, u_{2}\right) e^{-\left(\frac{u_{1}-x_{1}}{k}\right)^{2}-\left(\frac{u_{2}-x_{2}}{k}\right)^{2}} d u_{1} d u_{2},
$$

em que $\Delta_{1}$ é o quadrado de centro $M\left(x_{1}, x_{2}\right)$ e de lado $2 a$.

$\mathrm{O}$ autor concluiu que a integral acima converge para zero e assim demonstrou seu teorema, ou seja, que a função:

$$
\psi\left(x_{1}, x_{2}, k\right)=\frac{1}{k^{2} \pi} \iint_{P} f\left(u_{1}, u_{2}\right) e^{-\left(\frac{u_{1}-x_{1}}{k}\right)^{2}-\left(\frac{u_{2}-x_{2}}{k}\right)^{2}} d u_{1} d u_{2},
$$

tende para uma função somável $f\left(x_{1}, x_{2}\right)$ em todos os pontos do domínio mensurável $D$, exceto talvez nos pontos de um conjunto de medida nula.

Continuando o autor conclui que $\psi\left(x_{1}, x_{2}, k\right)$ tende uniformemente para a função contínua $f\left(x_{1}, x_{2}\right)$ no domínio fechado $D$. Como no caso das funções de uma variável deduz- 
se desta última conclusão a possibilidade de representar $f\left(x_{1}, x_{2}\right)$ no domínio $D$, por uma sucessão uniformemente convergente de polinômios.

Com esta conclusão Theodoro finalizou sua tese.

\section{Considerações:}

Ao debruçarmos sobre este trabalho, além da importância já citada no inicio deste texto, alguns aspectos são notórios para a época em que ele foi elaborado. Nota-se que dentre os vários autores por Theodoro mencionados ao longo do texto, alguns tiveram notável importância. A saber: Émile Borel $(1871$ - 1956) com seu trabalho sobre medida dos conjuntos; Henri Lebesgue (1875-1941) com seu estudo sobre integração; Karl Weierstrass (1815-1897) no tocante à representação efetiva das funções somáveis de uma variável pela integral de Weierstrass; Leonida Tonelli (1855-1946) referente ao estudo relativo aos polinômios de Landau de duas variáveis para a representação efetiva das funções somáveis de duas variáveis; e René-Louis Baire (1874-1932) por meio do trabalho sobre funções descontínuas, pois as funções de Baire enlaçam o teorema fundamental proposto por Theodoro Augusto Ramos em sua tese.

Estas teorias propiciaram a Theodoro Augusto Ramos (1895 - 1935) estudar a representação das funções somáveis de duas variáveis pela integral dupla de Weierstrass. Mostrou assim, que é natural basear a teoria das funções de variáveis reais sobre a simples noção de polinômio.

Embora esta tese não apresente uma lista de referências bibliográficas como conhecemos hoje, Theodoro mencionou ao longo do texto, os autores e obras utilizados por ele, sendo muitas vezes acrescentado o número da página e o ano da publicação. Neste sentido, no tocante às obras aludidas pelo autor, tornou-se possível construir a seguinte listagem com base no nome dos autores, obra e por vezes, ano e páginas. Foi possível identificar diretamente referências de Baire: Leçons sur les théories générales de l'Analyse [ t. 1. página 11] e Leçons sur les fonctions discontinues; Borel : Comptes-Rendus [ Journal de Jordan, 1912], Leçons sur la théorie des fonctions [2a edição, 1914, Note VI], Leçons sur les fonctions monogènes [1917], Leçons sur les fonctions de variables réelles [páginas 42, 54, 63, 74], Annales de l'Ecole Normale Supérieure [1895]; Gomes Teixeira: Curso de Analyse [ t. 1, 4a edição]; Goursat: Cours d'Analyse [t. 1, $3^{\mathrm{a}}$ edição, 1917 - páginas 454, 486]; J. Tannery: Introduction à la théorie des fonctions [t. 1, 2a edição], Leçons d'Algèbre et d'Analyse [t. 1. página 72]; Darboux: Annales de l'Ecole Normale Supérieure [1875]; H. Lebesgue: [ apud Cours d'Analyse de Goursat, t.1, 3a edição, 1917], Leçons sur l'intégration [ páginas 70, 71, 114], [apud E. Borel - Leçons sur les fonctions de variables réelles], Leçons sur les séries trigonométriques [página 14], Leçons sur l'intégration et la recherche des fonctions primitives [1904. página 124], Leçons sur les séries trigonométriques [1906. página 13], Annales de la Faculté des Sciences de Toulouse [ $3^{\text {ème }}$ série, t.1], [revista alemã Math. Ann. 61, 1905 apud M. Fréchet - Encyclopédie des Sciences Mathématiques, t. 3, v. $1,2^{\circ}$ fascículo, edição franceza, 191], Annali di Matematica pura ed applicata [serie III, tomo VII, 1902]; S. Pincherle [Lezioni di Calcolo, 1915]; Octacilio Novaes: Sobre o Calculo differencial (formula de Peano); S. Bernstein: [apud D'Adhémar - Leçons sur les principes de l'Analyse, t.2, 1913. (página 273)]; F. Riesz: Comptes-Rendus [ t. 148]; P. Montel: Leçons 
sur les séries de polynomes à une variable complexe [1910]; M. Potron: Sur une formule générale d'interpolation [Bulletin de la Societé Mathématique de France, 1906]; G. Vitali: Sui gruppi di punti e sulle funzione di variabili reali [Atti Accad. Torino, t. 43, 1907-1908] e G. Fubini: Sugli integrali multipli [Rendiconti Ac. dei Lincei, serie 5, t. 16, 1907, Roma].

Verifica-se que a teoria das funções de variáveis reais estudada por Theodoro desenvolveu-se após os trabalhos já citados de E. Borel, R. Baire e H. Lebesgue, pois o mesmo utilizou-se destes autores sistematicamente ao longo do texto. Merece destaque duas observações realizadas por Theodoro em que, primeiro: afirma que só podem ser representadas por sucessões de polinômios as funções de classe zero (funções contínuas) e as funções de classe 1 .

“Aliás, mediante um estudo previo das successões de funcções de Baire verifica-se effectivamente a possibilidade de edificar a theoria sobre esta noção. Convém observar que na definição de convergência simples a única noção nova que intervém é a de conjuncto de medida nulla, noção esta que pode ser adquirida independentemente da theoria geral da medida”. (RAMOS, 1918, p.4)

Segundo: Destaca-se que nosso personagem foi o primeiro a trabalhar a noção de convergência simples utilizando conjunto de medida nula, independentemente, da teoria geral da medida. Este fato pode ser averiguado em sua tese, sendo observado também no excerto abaixo transcrito do prefácio da mesma:

“F. Riesz (vide Comptes-Rendus, 1912) adoptou como ponto de partida a convergência simples de certas funcções discontinuas por elle denominadas de funcções simples, e edificou a parte essencial da theoria das funcções de variaveis reaes independentemente da theoria geral da medida, análogo estudo poderiamos fazer com os polynomios". (RAMOS, 1918, p.4)

Assim, em uma análise da tese de doutoramento produzida por Theodoro, podem ser destacados quatro pontos principais tomados pelo autor e que se refletem na compreensão do seu foco principal, que é também o principal teorema/resultado do trabalho realizado pelo autor. A saber: a questão abordada e referente à consideração das funções como limites de sucessões de polinômios; o estudo sobre teoria da medida; a construção da parte preliminar da teoria da integração das funções limitadas sem a intervenção da teoria geral da medida, utilizando a integral de uma função limitada com as propriedades da integral de Lebesgue e da integral de Borel; e finalmente, o principal resultado do trabalho que é dedicado à representação das funções somáveis de duas variáveis pela integral dupla de Weierstrass.

No tocante à demonstração do seu teorema principal pode-se perguntar a respeito de considerações sobre resultados utilizados na sua composição que foram estudados ou abordados primeiramente por Theodoro, demonstrados por outros autores e/ou ainda aquelas demonstrações dadas por esses outros autores e "moldadas" pelo personagem em estudo. 
Voltando-se os olhos para a demonstração deste teorema principal realizado por Theodoro, torna-se imperceptível e ao mesmo tempo impossível desvendar quais foram os resultados utilizados por este e advindos de outros autores, os por ele "moldados" ou aqueles genuinamente produzidos, uma vez que, neste sentido, nada é mencionado. Alargando a análise para toda a tese em questão, encontra-se análoga resposta para o questionamento acima. Quiçá mostram-se de maneira evidente os corolários, teoremas, proposições e até mesmo demonstrações advindas de outros autores e utilizadas pelo nosso personagem, uma vez que aparecem citadas ao longo do texto e podem ser observadas no original da tese.

$\mathrm{O}$ que deve ser ressaltado no resultado do teorema principal obtido pelo autor, cujo teor é inédito, é que este foi o primeiro a trabalhar a noção de convergência simples utilizando conjunto de medida nula, independentemente, da teoria geral da medida, contribuindo assim, significativamente, para o estudo da teoria das funções.

Nas palavras de Freire:

"Na sua These de doutorado, tratando da "representação effectiva das funcções sommaveis”, a uma variavel, fal-o Theodoro em relação á integral que representa uma funcção continua qualquer e que elle indica por $f(X, k)$. Havia, porém, Theodoro, pouco antes de fazer apparecer a sua These, recebido os Annaes de Toulouse onde verificou já ter sido tal representação obtida por Henri Lebesgue, seguindo, aliás, criterio bem differente do por elle adoptado. Por isso, conserva e apresenta a sua demonstração. A de Lebesgue surge como consequencia de dado criterio geral; a de Theodoro é directa. Constitue essa demonstração de Theodoro uma das bellas partes de sua These”. (FREIRE, 1936)

Deste modo, um estudo da tese de Theodoro Augusto Ramos mostra-se como relevante no tocante a aspectos matemáticos e históricos da época e posteriores. A notoriedade desta tese faz-se, principalmente, no tocante as contribuições para o desenvolvimento da análise matemática no país. Como já referenciado, esta foi defendida em uma escola de Engenharia - A Escola Politécnica do Rio de Janeiro -, um local que se destinava a graduar engenheiros sem o propósito de formar matemáticos ou professores de Matemática. Um lugar em que razoavelmente não se esperava que o ensino de Análise estivesse presente, e não estava.

\section{Bibliografia}

BAIRE, René-Louis. 1899. Sur les fonctions de variables réelles. Thèse présentées a la faculté des sciences de Paris pour obtenir la grade de docteur és Sciences Mathématiques, Milan: Imprimerie Bernardoni de C. Rebeschini \& C.

BONFIM, Sabrina Helena. 2013. Theodoro Augusto Ramos: um estudo comentado de sua tese de doutoramento. 2013. 124 f. Tese (doutorado) - Universidade Estadual Paulista Júlio de Mesquita Filho, Instituto de Geociências e Ciências Exatas. 
DOSSE, François. 2009. O Desafio Biográfico. Escrever uma Vida. Tradução de Gilson César Cardoso de Souza. São Paulo: Editora da Universidade de São Paulo.

FREIRE, Luis. 1936. A Obra Mathematica de Theodoro Ramos. Jornal do Commercio, Rio de Janeiro, 5 jul.

GAMA, Lélio Itapuambyra. 1965. Discurso do Professor Lélio Gama. Atas do $5^{\circ}$ Colóquio Brasileiro de Matemática, IMPA.

LEBESGUE, Henri. 1909. Sur les intégrales singulières. Annales de la faculté des sciences de Toulosse $3^{\mathrm{a}}$ série, tome I (1909), p. 25 - 117. (http://www.numdam.org/numdambin/feuilleter?id=AFST 1909 3_1_ ). Acesso em 30. mar. 2012.

RAMOS, Theodoro Augusto. 1918. Sobre as Funcções de Variaveis Reaes. Tese de doutorado. Escola Politécnica do Rio de Janeiro.

\section{Sabrina Helena Bonfim}

Universidade Federal de Mato Grosso do Sul UFMS - campus de Paranaíba - MS - Brasil

E-mail: sabrina_helenabonfim@yahoo.com.br

Marcos Vieira Teixeira

Departamento de Matemática - Universidade Estadual Paulista - UNESP - campus de Rio Claro -

SP - Brasil

E-mail: marti@rc.unesp.br 